\title{
校园环境质量监测实践课程的实施与体会
}

\author{
李广超”，杨伟华，袁兴程，贾文林，王海营，赵爽 \\ 江苏师范大学化学与材料科学学院, 江苏徐州 221116
}

摘要: 校园环境质量监测实践课程是一门实践性很强的综合性实践课程。课程目标既有知识与技能目标, 又有过程与方 法目标, 还有情感、态度和价值观目标。为了较好地达成课程目标, 既要制定合理的实施方案, 又要执行严格的实施过 程。指导教师要做好集体备课、课前动员、分组指导、成绩评定和课后总结的每一个环节。

关键词: 实践课程; 课程目标; 体会

中图分类号: G64; O6

\section{The Implementation and Experience of the Practical Course of Campus Environmental Quality Monitoring}

\author{
Guangchao Li *, Weihua Yang, Xingcheng Yuan, Wenlin Jia, Haiying Wang, Shuang Zhao \\ School of Chemistry and Materials Science, Jiangsu Normal University, Xuzhou 221116, Jiangsu Province, P. R. China.
}

\begin{abstract}
The practical course of campus environmental quality monitoring is a comprehensive practical course with strong practicality. The course objectives have not only the knowledge and the skill goal, but also have the process and the method goal, and also have emotional, attitudinal, and value goals. In order to better achieve the course goals, not only a reasonable implementation plan is formulated, but also a strict implementation process is performed. The instructors are responsible for collective lesson preparation, pre-class mobilization, group guidance, performance assessment and after-class summary.
\end{abstract}

Key Words: Practical course; Course objective; Experience

为培养大学生理论联系实际, 发现问题、分析问题和解决问题的能力, 特为应用化学专业、环 境工程专业和环境科学专业开设校园环境质量监测实践课程。该课程是在教师的指导下, 学生自主 进行的综合性实践课程。通过该课程的学习和实际操作, 不仅可以巩固所学的书本知识, 而且可以 将理论知识与实践相结合, 培养学生监测方案和实验方案设计能力, 巩固和提高实验操作基本技能, 初步掌握环境质量评价的方法。该课程还有助于提高实践育人环节的参与度和受益面 ${ }^{[1]}$, 凡是开设 了分析化学和环境监测课程的学生均可参与。实施时间可以设定在学生学习完环境监测课程后的两 周, 或者学完环境监测课程后的下一个学期, 一般为第 6 学期。

\section{1 课程目标与内容设计}

\section{1 课程目标}

(1) 知识与技能目标: 让学生学会设计水质监测、空气质量监测、声环境质量监测的方案, 学

收稿: 2020-01-27; 录用: 2020-03-17; 网络发表: 2020-03-30 
会布点方案设计和样品采集方法。

(2) 过程与方法目标: 培养学生实验方案设计、实验操作、数据处理以及对环境质量进行综合 分析和评价的能力。

(3) 情感、态度和价值观目标: 使学生养成实事求是的科学态度, 学会合作与分享, 懂得尊重 与欣赏, 形成良好的社会责任感和使命感。

\section{2 内容设计}

校园环境质量监测实践课程的主要内容及监测项目见表 1 。

表 1 校园环境质量监测实践的课程内容及测定项目

\begin{tabular}{ll}
\hline \multicolumn{1}{c}{ 内容 } & \multicolumn{1}{c}{ 测定项目 } \\
\hline 地表水环境质量监测 & 水温、 $\mathrm{pH}$ 、电导率、溶解氧、总 $\mathrm{N}$ 、总 $\mathrm{P} 、$ 六价铬、五日生化需氧量 $\left(\mathrm{BOD}_{5}\right)$ 等 \\
自来水质量检测 & $\mathrm{pH}$ 、电导率、溶解氧, 六价铬、总硬度、氯、总大肠菌群 \\
直饮水质量检测 & $\mathrm{pH}$ 、电导率、溶解氧, 总硬度、氯、总大肠菌群 \\
实验室污水检测 & $\mathrm{pH}$ 、电导率、溶解氧、总 $\mathrm{N} 、$ 总 $\mathrm{P}$ 、六价铬、化学需氧量 $(\mathrm{COD})$ \\
环境空气质量监测 & 校园空气中 $\mathrm{SO}_{2} 、 \mathrm{NO}_{2} 、 \mathrm{O}_{3} 、 \mathrm{PM}_{10}$ 等 \\
室内空气质量监测 & 办公室、体育馆、图书馆、实验室等场所的甲醛和总挥发性有机物 $(\mathrm{TVOC})$ \\
声环境质量监测 & 校园教学区和生活区的声环境质量 \\
\hline
\end{tabular}

\section{2 课程的实施过程}

课程实施过程主要包括：集体备课、课前动员、分组指导、成绩评定、课后总结。

\section{1 集体备课}

通常由教研室主任召集课程指导教师和实验室管理员针对该实践课程的具体实施过程进行的集 体备课，内容包括实践方案选择、学生分组和指导过程注意事项等。

(1) 教学实施方案。

教学过程中通常分为三种方案: 方案 1 是对校园内的地表水环境质量、环境空气质量、室内空 气质量以及声环境质量进行监测, 具体项目见表 2; 方案 2 是对校园的地表水环境质量进行监测, 并对自来水、教学区直接饮用水, 以及实验室污水进行检测, 具体项目见表 3; 方案 3 是分别对教 学区和生活区的环境空气质量以及室内空气质量进行监测, 具体项目见表 4。通常情况下, 每个教 学周期采取一种方案。

(2) 学生分组。

将一个班级随机分为 4 个小组, 每个小组先选择一项内容进行实践, 一周后与另一小组进行内 容和项目的调换。

(3) 实验室安排。

实验中心具有合格的环境监测实验室，可以开设水质监测、环境空气监测、噪声监测等方面的 实验内容, 为该课程的实施提供了条件保障。为防止实验过程中的相互影响, 将 4 个小组分别安排 在 4 个实验室中。

表 2 校园环境质量监测的内容和项目(方案 1)

\begin{tabular}{ll}
\hline 内容 & \multicolumn{1}{c}{ 测定项目 } \\
\hline 地表水环境质量监测 & 水温、 $\mathrm{pH}$ 、电导率、溶解氧、总 $\mathrm{N} 、$ 总 $\mathrm{P} 、$ 六价铬、 $\mathrm{BOD}_{5}$ \\
环境空气质量监测 & $\mathrm{SO}_{2} 、 \mathrm{NO}_{2} 、 \mathrm{PM}_{10}$ 等 \\
室内空气质量监测 & 甲醛、 $\mathrm{TVOC}$ \\
声环境质量监测 & 教学区和生活区昼间的声环境质量 \\
\hline
\end{tabular}


表 3 校园水环境质量监测的内容和项目(方案 2)

\begin{tabular}{ll}
\hline 内容 & \multicolumn{2}{c}{ 测定项目 } \\
\hline 地表水环境质量监测 & 水温、 $\mathrm{pH}$ 、电导率、溶解氧、总 $\mathrm{N}$ 、总 $\mathrm{P} 、$ 六价铬、 $\mathrm{BOD}_{5}$ \\
自来水质量检测 & $\mathrm{pH}$ 、电导率、溶解氧、六价铬、总硬度、氯含量 \\
直饮水质量检测 & $\mathrm{pH}$ 、电导率、溶解氧, 总硬度、氯含量、大肠菌群 \\
实验室污水检测 & $\mathrm{pH}$ 、电导率、溶解氧、总 $\mathrm{N} 、$ 总 $\mathrm{P} 、$ 总铬、 $\mathrm{COD}$ \\
\hline
\end{tabular}

表 4 校园环境空气质量监测的内容和项目(方案 3)

\begin{tabular}{ll}
\hline \multicolumn{1}{c}{ 内容 } & \multicolumn{1}{c}{ 测定项目 } \\
\hline 教学区环境空气质量监测 & $\mathrm{SO}_{2} 、 \mathrm{NO}_{2} 、 \mathrm{CO} 、 \mathrm{O}_{3} 、 \mathrm{PM}_{10}$ 等 \\
生活区环境空气质量监测 & $\mathrm{SO}_{2} 、 \mathrm{NO}_{2} 、 \mathrm{CO} 、 \mathrm{O}_{3} 、 \mathrm{PM}_{10}$ 等 \\
办公室、实验室室内空气质量监测 & 甲醛、 $\mathrm{TVOC}$ \\
体育馆、图书馆室内空气质量监测 & 甲醛、TVOC \\
\hline
\end{tabular}

\section{2 课前动员}

在进行校园环境质量监测实践课程实施前召开的动员会议, 通过动员让学生明确实践课程的重 要性, 同时让学生明确课程的任务和要求、考核方法、学生分组情况及组长确定等。

\section{3 分组指导}

每个小组领到任务后, 指导教师进行分组指导。一名教师可以指导一组或两组学生。指导内容 主要包括: 依据环境监测相关知识并查阅资料, 按照相关国家标准和监测技术规范, 科学合理制定 监测方案(包括采样点的布设、样品的采集和预处理方法等); 依据要测定项目的国家标准方法, 制 定实验方案; 正确规范地测定选定的项目, 并根据《地表水环境质量标准》(GB3838-2002)对校园 地表水环境质量进行评价, 根据《生活饮用水卫生标准》(GB5749-2006)对校园自来水和直饮水质 量进行评价, 根据《环境空气质量标准》(GB3095-2012)进行校园环境空气质量评价, 根据《室内 空气质量标准》(GB/T 18883-2002)进行校园室内空气质量评价, 根据《声环境质量标准》(GB 30962008)进行校园内教学区和生活区昼间的声环境质量评价。

\section{4 成绩评定}

从学生的监测方案(10 分)、实验方案(10 分)、实验操作(20 分)、数据处理(20 分)、评价报告(20 分)、学习态度(10 分)、反思报告(10 分)等多维度进行成绩评定。每组学生的各项成绩由指导教师集 体评定。

\section{5 课后总结}

课程结束后召开总结会。每个小组指派一名学生代表在总结会上发言, 主要汇报学习该课程的 收获、体会及反思等。指导教师针对学生在监测方案、实验方案、实验操作、数据处理、评价报告 等方面值得肯定的地方以及存在的问题进行详细总结。

\section{3 校园环境质量监测实践的实施效果与体会}

\section{1 实施效果}

对一个班级的成绩评定结果进行统计, 监测方案、实验方案、学习态度和反思报告四项总分均 为 10 分的项目得分统计结果见图 1, 实验操作、数据处理、评价报告三项总分均为 20 分的项目得 分统计结果见图 2。从图 1、图 2 中可以看出, 对于监测方案、实验方案、数据处理和评价报告来说, 同一小组是一样的, 因此同一小组的不同学生得分相同, 但小组之间存在差异。对于实验操作、学 习态度和反思报告来说, 全班学生存在差异。最高得分达到满分, 最低得分为该项目分数的 $60 \%$ 。 从图 1、图 2 中还可以看出, 学生在实验操作、反思报告和学习态度方面存在较大差异。 


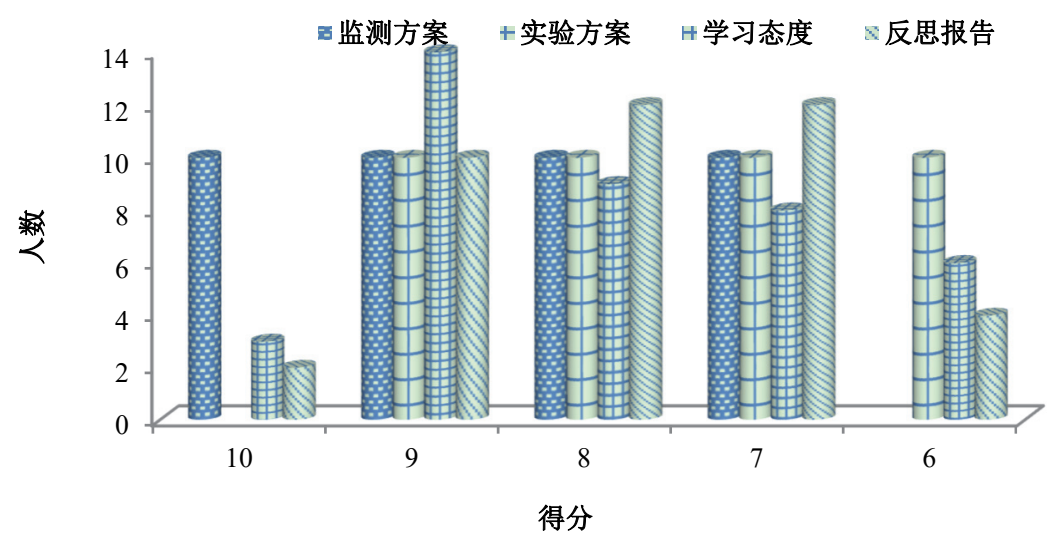

图 1 项目分数为 10 分的统计结果

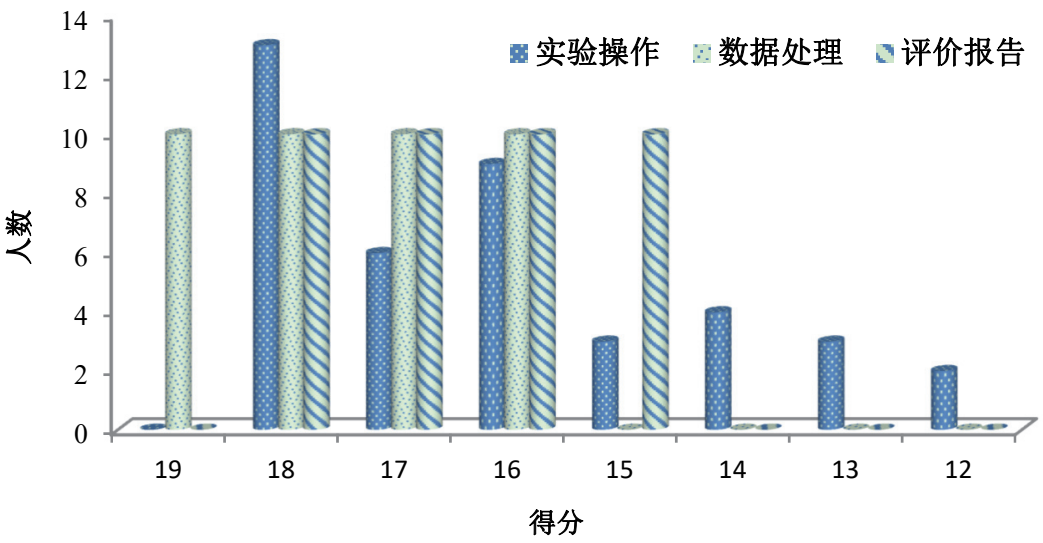

图 2 项目分数为 20 分的统计结果

选取一个班级的成绩评定结果进行项目达成度评价和课程达成度评价。评价项目达成度为某项 目的平均得分与该项目总分的比值, 如监测方案平均分为 8.5 分, 该项目总分 10 分, 于是该项目的 达成度为 0.850 。课程达成度为课程的平均得分与课程总分(100 分)的比值, 评价结果见表 5 。若课 程的目标达成度设定为 0.800 , 而实际课程达成度为 0.820 , 表明该课程的教学效果达到了课程目标 要求。从评价项目的达成度来看, 监测方案、实验操作、数据处理、评价报告和学习态度六项的达 成度大于或等于 0.800 , 达成度较高, 表明学生这几方面的知识和能力达到了课程要求。而实验方案 和反思报告的达成度分别为 0.750 和 0.785 , 达成度较低, 表明学生的实验方案设计能力有待于进一 步提高，在情感、态度和价值观目标方面也有待于进一步加强。

表 5 评价项目达成度及课程目标达成度

\begin{tabular}{ccccccccc}
\hline 项目 & 监测方案 & 实验方案 & 实验操作 & 数据处理 & 评价报告 & 学习态度 & 反思报告 & 课程 \\
\hline 达成度 & 0.850 & 0.750 & 0.805 & 0.875 & 0.825 & 0.800 & 0.785 & 0.820 \\
\hline
\end{tabular}

总之, 校园环境质量监测实践课程的实施取得了良好的效果。一是较好地实现了课程目标, 使 学生体验了监测方案的制定和实验方案设计, 初步掌握了环境质量评价的基本方法, 初步具备撰写 环境质量评价报告的能力, 同时学生的实验操作技能也有一定程度的提高。二是在课程进行过程中, 小组与小组之间, 同学与同学之间进行有效沟通交流, 使学生认识到团队协作的重要性, 增强了团 
队协作的意识。三是通过个人反思报告和小组代表的发言, 使每位学生认识到自己在课程实施过程 中的收获, 学会反思自己在课程实施过程中存在的不足, 这对学生的学习、成长和工作都十分重要。

\section{2 体会}

(1) 课程组织与实施过程要做到 “两早两好”。

“两早” 是指集体备课要早, 课前动员要早。

(1) 集体备课要早。集体备课要在课程实施前两至三周进行, 以便于确定实施方案后, 实验管理 人员可以有充足时间准备仪器和试剂, 防止在课程进行期间, 学生找不到试剂而影响课程的顺利实 施。

(2) 课前动员要早。课前动员最好提前一到两周进行, 让学生有充足时间查阅资料, 在课程开始 前就明确任务和要求, 并能早点制定好监测方案, 设计好实验方案, 从而保证学生有充足时间进行 项目的测定和评价报告的撰写。

“两好” 是指学生分组要好, 教师指导要好。

(1) 学生分组要好。在进行分组时既要考虑学生的学习成绩, 又要兼顾男女生比例。要防止一个 小组中全是学习成绩比较差的学生, 也要尽可能使每个小组的男女生人数均衡。

(2) 教师指导要好。课程指导教师要在监测方案制定、实验方案设计、实验操作、数据处理、评 价报告撰写等方面进行全方位指导。在指导学生进行监测方案制定和实验方案设计时, 不是简单地 告诉学生那样不对, 或这样不好, 而是要让学生知其然, 又知其所以然。在指导实验操作时, 更要 指导到位。不仅要耐心细致, 而且要严格规范。要时时观察学生的实验操作过程, 若发现学生的实 验操作不规范, 应及时纠正, 反复练习, 达到熟练掌握。在课程实施过程中, 指导教师要有强的责 任心, 特别要杜绝指导教师在整个课程中对学生不指导不考察, 布置好任务后, 就不闻不问了, 到 课程结束时让学生随便交个报告, 再随便给学生打个分就行了。这样不仅不能够实现课程目标, 也 会让学生感觉到该门课程是水课，随便混混就可以了。

(2) 校园环境质量监测的内容与项目的确定要考虑 “两个条件”。

在确定校园环境质量监测的内容和项目时, 一是要考虑实验室是否具备所选取监测项目的监测 设备和其他实验条件，以及在课程实施期间的有限时间内能否顺利完成。二是要考虑国家或地方标 准中是否含有课程确定的内容和项目, 以便于准确地进行评价。方案 2 中的实验室污水检验项目, 其实没有相应的标准, 只能参考污水综合排放标准(GB 8978-1996)进行评价。另外, 实验室综合污 水的排放量很不均匀, 污水成分与开设的实验项目密切相关, 这样就为实验室污水检测项目的选择、 测定和评价带来很大困难, 因此实验室综合污水检测不太适合该课程的实施内容。如果一定要进行 该项内容，最好选择某实验室的回收废液进行测定。

\section{4 结语}

校园环境质量监测实践课程是一门实践性很强的综合性实践课程。课程目标既有知识与技能目 标, 又有过程与方法目标, 还有情感、态度和价值观目标。初步实施效果显示, 学生不仅在监测方 案的制定、实验方案设计、实验基本操作、环境质量评价报告撰写等方面的能力得到了提高, 而且 认识到团队协作的重要性, 增强了团队协作的意识。在课程开设过程中体会到, 为了能很好地达成 课程目标, 一是必须具备环境监测的实验条件, 二是要制定合理的实施方案, 三是要有严格的实施 过程。要做好集体备课、课前动员、分组指导、成绩评定和课后总结的每一个环节, 特别要重视指 导教师在课程实施过程中的作用。 